Article

\title{
Organic Solid-State Tri-Wavelength Lasing from Holographic Polymer-Dispersed Liquid Crystal and a Distributed Feedback Laser with a Doped Laser Dye and a Semiconducting Polymer Film
}

\author{
Minghuan Liu ${ }^{1,2}$, Yonggang Liu ${ }^{1}$, Zenghui Peng ${ }^{1}$, Shaoxin Wang ${ }^{1}$, Qidong Wang ${ }^{1}$, \\ Quanquan $\mathrm{Mu}^{1}$, Zhaoliang Cao ${ }^{1}$ and Li Xuan ${ }^{1, *}$ \\ 1 State Key Laboratory of Applied Optics, Changchun Institute of Optics, Fine Mechanics and Physics, \\ Chinese Academy of Sciences, Changchun 130033, China; liuminghuan13@mails.ucas.ac.cn (M.L.); \\ liuyonggang@ciomp.ac.cn (Y.L.); peng@ciomp.ac.cn (Z.P.); wangshaoxin@ciomp.ac.cn (S.W.); \\ wangqidong@ciomp.ac.cn (Q.W.); muquanquan@ciomp.ac.cn (Q.M.); caozlok@ciomp.ac.cn (Z.C.) \\ 2 University of Chinese Academy of Sciences, Beijing 100049, China \\ * Correspondence: xuanli@ciomp.ac.cn
}

Academic Editors: M. Blanca Ros, Raquel Gimenez and Teresa Sierra

Received: 24 March 2017; Accepted: 3 May 2017; Published: 7 May 2017

\begin{abstract}
Organic solid-state tri-wavelength lasing was demonstrated from dye-doped holographic polymer-dispersed liquid crystal (HPDLC) distributed feedback (DFB) laser with semiconducting polymer poly[-methoxy-5-(2'-ethyl-hexyloxy)-1,4-phenylene-vinylene] (MEH-PPV) and laser dye [4-(dicyanomethylene)-2-methyl-6-(p-dimethylaminostyryl)-4H-pyran] (DCM) by a one-step holography technique, which centered at $605.5 \mathrm{~nm}, 611.9 \mathrm{~nm}$, and $671.1 \mathrm{~nm}$. The temperature-dependence tuning range for the tri-wavelength dye-doped HPDLC DFB laser was as high as $8 \mathrm{~nm}$. The lasing emission from the 9th order HPDLC DFB laser with MEH-PPV as active medium was also investigated, which showed excellent s-polarization characterization. The diffraction order is 9 th and 8 th for the dual-wavelength lasing with DCM as the active medium. The results of this work provide a method for constructing the compact and cost-effective all solid-state smart laser systems, which may find application in scientific and applied research where multi-wavelength radiation is required.
\end{abstract}

Keywords: holographic polymer-dispersed liquid crystal; distributed feedback; tri-wavelength lasing; tuning; diffraction order

\section{Introduction}

Organic solid-state lasers (OSSLs) have attracted substantial scientific interest since they are compact, cost-efficient, operate at a low threshold, and have wide spectral covering and tunability [1-4]. Moreover, OSSLs have the potential to achieve electrical injection [5], which makes them smart and promising laser sources for spectroscopy [6], integrated photonics [7], and sensing [8].

OSSLs usually use organic laser dye-doped medium [9] or semiconducting polymer film [10] as active medium. The cavity configuration such as distributed feedback (DFB) [11], distributed Bragg reflector (DBR) [12], and micro-cavity [10] was used to provide positive feedback and lasing mode selection. The DFB configuration provides excellent mode selection and operates with a low threshold among those configurations [2]. The DFB configuration can be fabricated by molding [13] and imprinting [11] process. In addition, the holographic polymer-dispersed liquid crystal (HPDLC) grating can also provide well-defined DFB configuration via polymer-induced phase separation (PIPS) [14]. Moreover, the HPDLC grating show advantages over those molding and imprinting process for it is cost-effectiveness, ease of fabrication, and mass production. 
Multi-wavelength OSSLs are useful and promising devices, especially in the field of communications [15] and lab-on-a-chip [16], since multi-wavelength lasing can be achieved with one smart laser. To achieve this goal, Diao et al. reported the dual-wavelength lasing from the semiconducting film and the doped dye using the HPDLC as oscillation cavity [17]. Zhang et al. demonstrated the surface-emitting dual-wavelength laser from a blended gain layer using two semiconducting polymer [18]. However, they just used two kinds of gain medium and cannot provide insights for guidance. The tri-wavelength lasing was not demonstrated for OSSLs from the HPDLC as oscillation cavity until now.

In previous work, we demonstrated that the organic holographic polymer-dispersed liquid crystal and a distributed feedback laser from different diffraction orders, and we believed that the combination of the diffraction order and LC provide a more intriguing feature to the HPDLC DFB laser [19]. In this study, a tri-wavelength lasing from a holographic polymer-dispersed liquid crystal and a distributed feedback laser with dye-doped HPDLC and a semiconducting polymer film as an active medium was demonstrated - and to the best of our knowledge, for the first time. The semiconducting polymer film was spin-coated onto a glass substrate and sandwiched between the glass substrate and the HPDLC grating film. The lasing emission from the 9 th diffraction order for the HPDLC DFB laser was investigated. In addition, the laser dye [4-(dicyanomethylene)-2-methyl-6-(p-dimethylaminostyryl)-4H-pyran] (DCM) was doped into the HPDLC grating. The laser dye DCM and semiconducting polymer film were both encapsulated into the device to prevent photo-induced oxidation and degradation. A dual-wavelength lasing was also achieved from a diffraction order different from DCM. The experimental results demonstrated that multi-wavelength lasing occurs when there are multi-cavity modes in the gain spectra. The laser device, which is lightweight, smart, cost-effective, and easy to fabricate, and enjoys tri-wavelength operation and tunability, is promising in the fields of sensing and spectroscopy.

\section{Materials and Methods}

\subsection{Sample Preparation}

The poly[-methoxy-5-(2'-ethyl-hexyloxy)-1,4-phenylene-vinylene] (MEH-PPV) film was used as an active medium layer. The MEH-PPV (Polymer Light Technology) was dissolved in tetrahydrofuran (THF) by weight ratio at $0.6 \mathrm{wt} \%$. The solutions were stirred for $72 \mathrm{~h}$ to ensure sufficient dissolution. A drop of MEH-PPV solution was injected on a piece of deionized pre-clean glass substrate for spin-casting. The thickness of the MEH-PPV film was controlled by varying the spin speed and measured by a surface profiler (KLA Tencor P-16+). The thickness was controlled at $75 \mathrm{~nm}$ for good lasing operation [20]. All experiments were performed under the same ambient circumstances.

For holographic polymer-dispersed liquid crystal (HPDLC) distributed feedback (DFB) laser fabrication, the HPDLC grating film was recorded onto the MEH-PPV film via holography photo-induced polymerization [14]. The holographic medium mixture for HPDLC mainly contained acrylate monomers (dipentaerythritol hydroxyl pentaacrylate (DPHPA, Aldrich, $29.4 \mathrm{wt} \%$, Shanghai, China) and phthalicdiglycoldiacrylate (PDDA, Eastern Acrylic Chem, $29.4 \mathrm{wt} \%$, Shandong, China)) and nematic liquid crystals (TEB-30A, $n_{0}=1.522, \Delta n=0.170$, Silichem, $29.4 \mathrm{wt} \%$ ). Crosslinking monomer N-vinylpyrrolidone (NVP, Aldrich, $9.8 \mathrm{wt} \%$, Shanghai, China) was also added to dilute the mixture. Rose Bengal (RB, Aldrich, 0.5 wt \%, Shanghai, China) and N-phenylglycine (NPG, Aldrich, $1.5 \mathrm{wt} \%$, Shanghai, China) was used as photo-initiator and co-initiator, respectively [19]. The laser dye 4-(dicyanomethylene)-2-methyl-6-(p-dimethylaminostyryl)-4H-pyran (DCM, Aldrich, $0.5 \mathrm{wt} \%$, Shanghai, China) was added in the mixture for dye-doped HPDLC DFB laser fabrication [17]. The mixture, which was stirred with a magnetic bar for $48 \mathrm{~h}$ for a homogeneous material system, was injected into an empty glass cell by capillary action in a darkroom. The empty cell was made with two pieces of glass substrate. One had a spin-cast MEH-PPV film, and the other was a pure glass substrate. The thickness of the cell was controlled by spacers at $9 \mu \mathrm{m}$, which was thick enough for the doping DCM laser dye to absorb enough excitation energy for lasing oscillation buildup when photo 
pumping. The refractive index of the polymer-dispersed liquid crystal (PDLC, (the LC with random alignment and the polymer)) was 1.543 at $589.0 \mathrm{~nm}$, which was confirmed by an Abbe refractometer (2WA, Kernco, El Paso, TX, USA). The refractive index of MEH-PPV film was 2.0 at $589.0 \mathrm{~nm}$ [21].

\subsection{HPDLC Grating Fabrication}

The unslanted transmittance HPDLC grating film was photo-cured by illuminating the cell for three minutes by two s-polarized continuous frequency doubled neodymium-doped yttrium aluminum garnet $\left(\mathrm{Nd}^{3+}\right.$ :YAG) laser beams (New Industries Optoelectronics, Changchun, China), as shown in Figure 1a [20]. The recording beam was expanded and filtered to ensure uniform illumination. A variable optical attenuator was inserted to the beam path to regulate the beam intensity. The coherent laser beams pass through the pre-polymer mixture first when fabricating the HPDLC grating as shown in Figure 1a. The energy will be absorbed strongly when the MEH-PPV film faces the coherent laser beams. Therefore, the interference field cannot be built up effectively. The HPDLC film can also protect the MEH-PPV film from photo-induced oxidation and degradation [22]. The period of the HPDLC grating film is determined by

$$
\Lambda=\frac{\lambda_{r e c}}{2 \sin (\theta)}
$$

where $\Lambda$ is the period of the HPDLC grating, $\lambda_{\text {rec }}=532 \mathrm{~nm}$ is the recording laser wavelength in vacuum, and $\theta$ is half of the intersection angle between two recording beams. The intersection angle can be changed to obtain different grating periods in experiments. Figure $1 \mathrm{~b}$ depicts the device structure without DCM dye doping. Figure $1 b, c$ is in agreement with each other and they are complementary. The sample is transversely excited in both Figure 1b,c. Figure $1 b$ demonstrates mainly in the lasing oscillation buildup mechanism and Figure 1c demonstrates the collection of the lasing signal. The lasing signal is coupled out via grating coupling as shown in Figure 1c. Details can be found in Section 3.2.

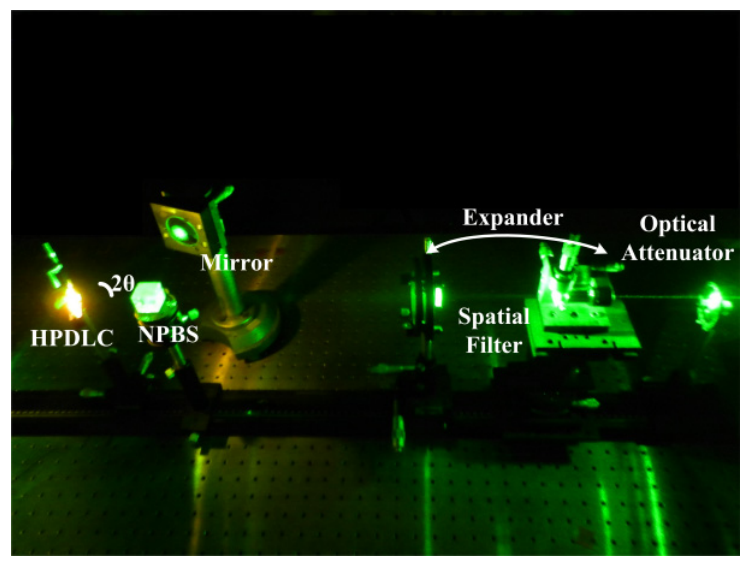

(a)

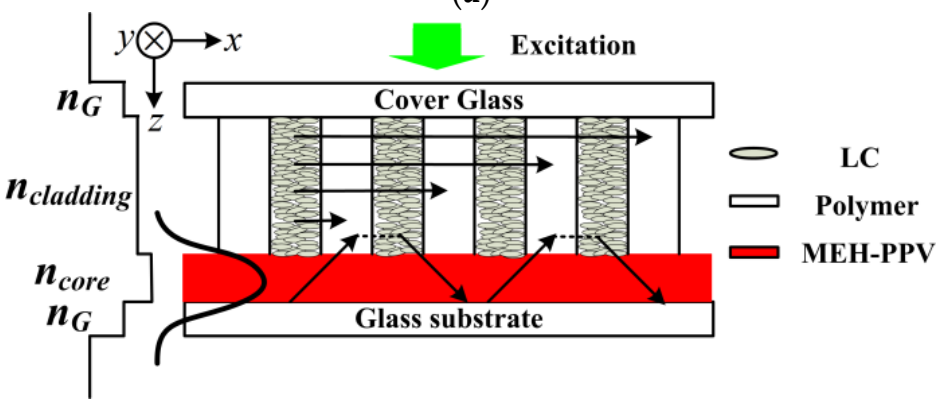

(b)

Figure 1. Cont. 


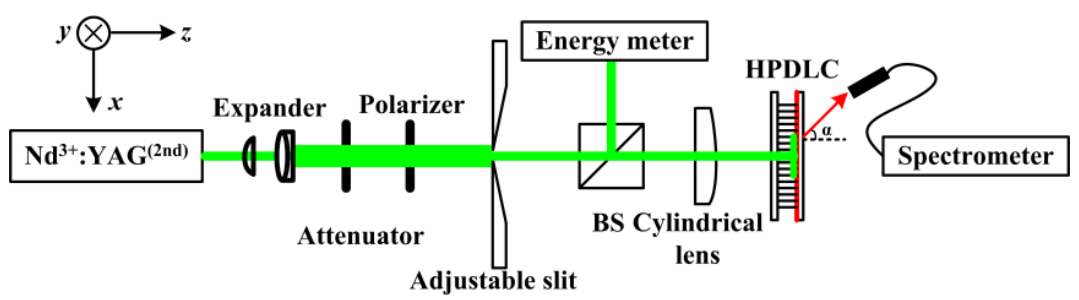

(c)

Figure 1. (a) Experimental schematic setup for holographic polymer-dispersed liquid crystal (HPDLC) and distributed feedback (DFB) film fabrication. (b) Schematic illustration of HPDLC DFB laser device structure and (c) an HPDLC DFB laser lasing characterization experimental diagram.

\subsection{Lasing Characterization}

For lasing characterization, the samples were transversely optical excited by a frequency doubled Q-switched $\mathrm{Nd}^{3+}$ :YAG pulsed laser, which delivered $10 \mathrm{~ns}$ pump pulses at a repetition rate of $10 \mathrm{~Hz}$ and an excitation wavelength of $532 \mathrm{~nm}$ (New Industries Optoelectronics), as shown in Figure 1c. The output lasing signal was collected with a fiber-coupled grating spectrometer, which possessed a resolution limit at $0.23 \mathrm{~nm}$ (Sofn Instruments, Beijing, China). The excitation energy was monitored by redirect part of the incident energy into an energy meter via a non-polarized beam splitter (BS) in real time. The pump laser beam was expanded and collimated, and only the central part was selected in order to ensure uniform pumping. Then, the excitation beam was reshaped with a cylinder lens $(\mathrm{f}=200 \mathrm{~mm})$ to produce a $3 \mathrm{~mm}$ by $1 \mathrm{~mm}$ excitation beam at a $0^{\circ}$ angle with respect to the normal of the sample. Moreover, a polarizer was used to set the incident polarization. The variable optical attenuator was used to regulate the pump energy to investigate output emission intensity as a function of excitation energy.

\section{Results and Discussion}

\subsection{Spectroscopic Characterization}

The pure film spectrum indicates the essential electronic and vibrational structure properties of an organic material. An active medium can be used appropriately by understanding the essential electronic and vibrational structure. Generally, the excitation laser wavelength should match the maximum absorbance wavelength well in order to achieve the population inversion effectively. In addition, the cavity modes should be involved in the gain spectrum of the active medium for lasing emission operations according to laser principles. Moreover, the lasing mode should be optimized to the position where the active medium is a four-level system for low-threshold operation [2,23]. Figure 2 shows the spectroscopic properties of the active medium MEH-PPV film and the laser dye DCM. The absorbance spectra of the spin-coating MEH-PPV film and the laser dye DCM were performed by a UV-3101PC (SHIMADZU, Shanghai, China) UV-VIS-NIR spectrometer. For spectroscopic characterization, the laser dye DCM was doped into DPHPA/NVP (6:1 by weight) by a weight ratio at $0.5 \%$ and then injected into a clean glass cell by a capillary action. The photoluminescence (PL) spectra were performed by F-7000FL (Hitachi, Shanghai, China) spectrometer, and the excitation wavelengths were selected at $500 \mathrm{~nm}$ and $480 \mathrm{~nm}$ to match the peak absorbance of MEH-PPV and DCM, respectively. The broad absorbance spectra show a single peak locating at $500.8 \mathrm{~nm}$ and $480 \mathrm{~nm}$ in the visible spectral band for MEH-PPV and DCM respectively, where the absorbance is the maximum. The full width at half maximum (FWHM) values of the absorbance spectra are $90 \mathrm{~nm}$ and $120 \mathrm{~nm}$ for DCM and MEH-PPV, respectively. The absorbance spectra confirm that the absorbance are sufficient for both of the active media when photo-pumping the sample at $532 \mathrm{~nm}$. The peaks of the PL spectra represent an electronic and vibrational structure, the 0-0 and 0-1 emission band. The $\mathrm{S}_{0-0}$ represents the transition from the bottom of the first excited singlet state to the bottom of the ground state, which corresponds to a three-level system. While the 
$\mathrm{S}_{0-1}$ represents the transition from the bottom of the first excited singlet state to the first vibration level of the ground state, the molecules will then reach the bottom of the ground state via thermal relaxation, which corresponds to a four-level system. The $S_{0-0}$ and $S_{0-1}$ peaks of the PL spectra are centered at $590 \mathrm{~nm}$ and $634 \mathrm{~nm}$ for MEH-PPV, and they are $580 \mathrm{~nm}$ and $610 \mathrm{~nm}$ for DCM, respectively. As a result, the large stokes shift (the spectral range between the absorbance peak and the fluorescence peak) of DCM and MEH-PPV red-shifts their fluorescence emission from absorbance and makes them a good candidate to be used as active medium.

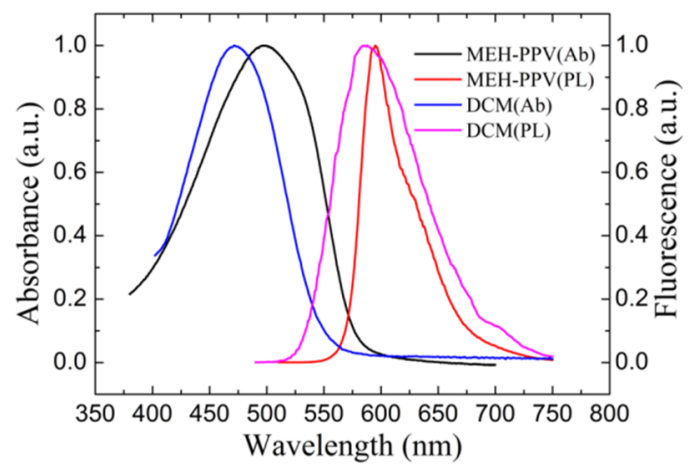

Figure 2. Spectroscopic characterization, e.g., the absorbance and fluorescence spectra of the active medium MEH-PPV and DCM. The fluorescence spectrum was collected with continuous excitation at 500 and $480 \mathrm{~nm}$ for MEH-PPV and DCM, respectively.

\subsection{The Mechanism of HPDLC DFB Laser}

According to Kogelnik's coupled wave theory about the DFB laser [24], the lasing wavelength $\lambda_{\text {las }}$ in vacuum of the HPDLC DFB laser [20] can be expressed as

$$
\lambda_{\text {las }}=\frac{2 n_{e f f} \Lambda}{m},
$$

where $n_{\text {eff }}$ is the effective refractive index of the lasing mode, $\Lambda$ is the HPDLC grating period, and $\mathrm{m}$ is the diffraction order. Equation (2) demonstrates that the HPDLC grating period and the diffraction order can be changed so as to select a variable lasing wavelength from the HPDLC DFB laser. For a constant diffraction order, the tunability can be achieved by varying the grating period. The tunable range is the gain spectrum in which the lasing works [25].

The light field will be amplified when it travels through the HPDLC grating/MEH-PPV film/glass substrate waveguide configuration during the excitation process for the HPDLC DFB laser. The refractive index of the MEH-PPV film is higher than the HPDLC grating film and the glass substrate, so the light field is mainly confined in the MEH-PPV film as shown in Figure 1b. In addition, the evanescent light field travels into the HPDLC grating and travels a distance in the HPDLC grating via Goos-Haenchen shift [26]. (The Goos-Haenchen shift occurs in the case of total internal refection. When the total internal reflection happens, the incident light wave from the high refractive index medium at the medium boundary will travel into the low refractive index medium at the depth of one $\lambda$. There is no power transport into the low refractive index medium. The distance between the entrance and the exit of the incident light wave is about half of one $\lambda$, which is the Goos-Haenchen shift.) As a result, the light field is compressed and selected by the HPDLC grating. Then, the evanescent light field travels back into the MEH-PPV film. Thus, the light field will be further amplified by the MEH-PPV film and further compressed by the HPDLC grating. In such a process, the positive feedback builds up and the laser eventually works, as shown in Figure $1 b$.

Figure 3 demonstrates lasing properties of the 9th order HPDLC DFB laser. The grating period was selected at $1.72 \mu \mathrm{m}$ to achieve the 9th order HPDLC DFB laser. The line width of the fluorescence of MEH-PPV decreases with excitation energy until the lasing threshold. The central wavelength of 
the lasing emission is $611.9 \mathrm{~nm}$, with a full width at half maximum (FWHM) at $0.64 \mathrm{~nm}$, as shown in Figure 3a. The spectrum is compressed intensely in comparison with the fluorescence spectrum as shown in Figure 2, which confirms the excellent spectral compression and selectivity of the HPDLC grating. The quality factor $Q(Q=\lambda / \Delta \lambda)$ is as high as 956. The single peak and the quality factor confirm the good lasing and single longitudinal mode operation. The effective refractive index of the $611.9 \mathrm{~nm}$ lasing mode is 1.60. A polarizer was placed before the detector system to investigate the polarization of the emission beams. The emission intensity as a function of the polarizer axis rotation angle is shown in Figure $3 b$. The square points are experimental while the solid line is a cosine fitting of the experimental data. The polarization degree $\left(\left(I_{\|}-I_{\perp}\right) /\left(I_{\|}+I_{\perp}\right) \times 100 \%\right)$ is as high as $99 \%$, which confirms the excellent s-polarization property of the emission beams.

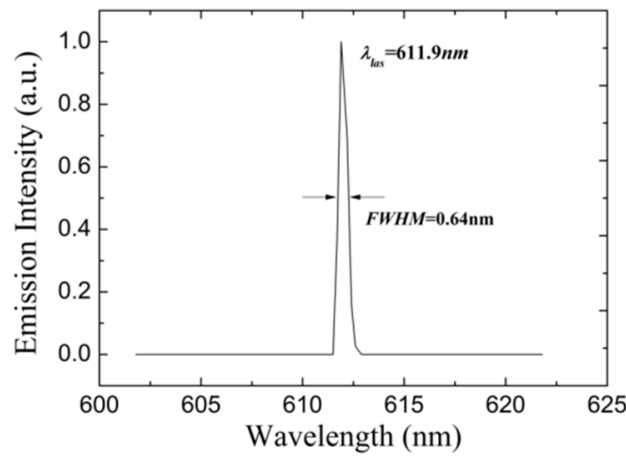

(a)

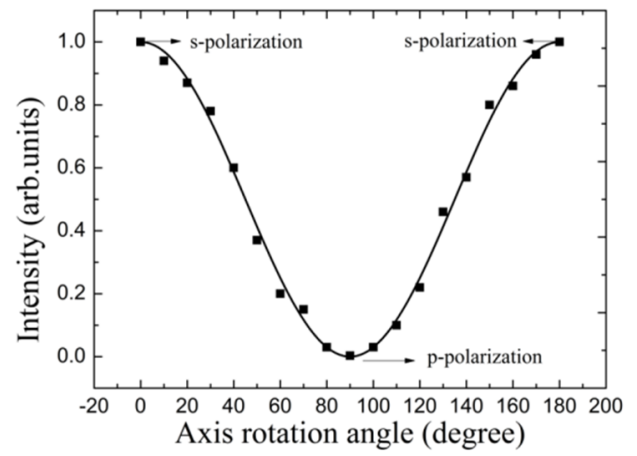

(b)

Figure 3. Lasing property of the 9th order HPDLC DFB laser. (a) Lasing spectra with the grating period selected at $1.72 \mu \mathrm{m}$ and (b) the emission intensity as a function of the polarizer axis rotation angle.

For the diffraction order $\mathrm{m}>1$, the lasing radiation is coupled out of the waveguide by an angle $\alpha$ relative to the HPDLC DFB laser surface normal via grating coupling [27-30] as shown in Figure 1c. The guided mode travelling in the HPDLC DFB laser [28] can be expressed as

$$
k^{2}=\left(n G_{0}-k_{0} \sin \alpha\right)^{2}
$$

where $k$ is the wavenumber of the light travelling in the MEH-PPV film, $n$ is an integer, $G_{0}$ is the wavenumber of the HPDLC grating $\left(G_{0}=2 \pi \Lambda^{-1}\right)$, and $k_{0}$ is the wavenumber of the light in vacuum. Figure 4 depicts the emission pattern of the 9th order HPDLC DFB laser. The emission angles are $32,62,118$, and $148^{\circ}$, which is in good agreement with the theoretical calculations performed with Equation (3).

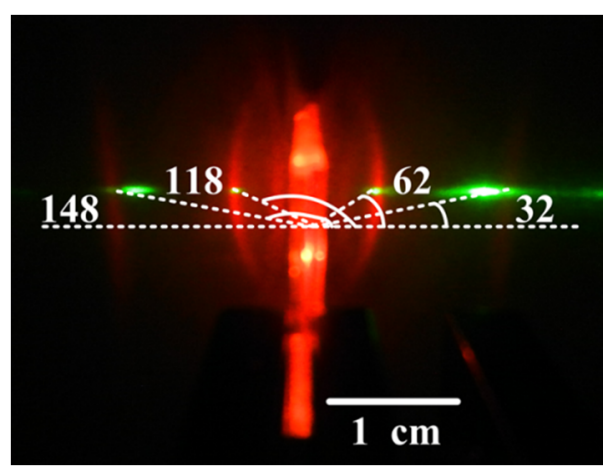

Figure 4. The emission pattern of the 9th order HPDLC DFB laser collected by a digital camera with the excitation energy at $20 \mu \mathrm{J} /$ pulse. 


\subsection{Tri-Wavelength Lasing from Dye-Doped HPDLC DFB Laser}

The multi-wavelength lasing emission operates when cavity modes exist within the gain spectrum [2,23]. It is confirmed that the DCM has a broad fluorescence spectrum as shown in Figure 2. Moreover, the lasing emission reported for DCM was from 574 to $685 \mathrm{~nm}$ with different period HPDLC grating as oscillation cavity $[17,31,32]$. Therefore, the dual-wavelength lasing emission operates between two adjacent diffraction orders when two cavity modes exist within the gain spectrum of the laser dye DCM according to Equation (2). In previous work, we reported that the lasing emission for MEH-PPV ranged from 590 to $657 \mathrm{~nm}$; as a result, the dual-wavelength lasing cannot operate in this study [19]. The DCM dye-doped HPDLC DFB laser with a grating period selected at $1.72 \mu \mathrm{m}$ was fabricated to achieve the tri-wavelength lasing emission. Figure 5a shows the lasing spectra of the dye-doped HPDLC DFB laser. Thanks to the distributed feedback mechanism for both active media MEH-PPV and DCM, the lasing emission was collected from the sample edge at one time. The lasing emission centers at $605.0 \mathrm{~nm}, 611.9 \mathrm{~nm}$, and $677.1 \mathrm{~nm}$ as shown in Figure 5a. The good lasing spectra indicate the reliability of the tri-wavelength HPDLC DFB laser. The $605.0 \mathrm{~nm}$ and $677.1 \mathrm{~nm}$ lasing was delivered from DCM, confirming that the theory is reasonable. The $611.9 \mathrm{~nm}$ lasing was delivered from MEH-PPV, which confirmed that the lasing from DCM and MEH-PPV propagate independently. In addition, the diffraction order for the $605 \mathrm{~nm}, 611.9 \mathrm{~nm}$, and $677.1 \mathrm{~nm}$ lasing is 9th, 9th, and 8th, respectively. The effective refractive index of the $605.0 \mathrm{~nm}, 611.9 \mathrm{~nm}$, and $677.1 \mathrm{~nm}$ lasing is $1.58,1.60$, and 1.572, respectively. Figure $5 \mathrm{~b}$ shows the dependence of output emission intensity to excitation energy of the tri-wavelength laser. The lasing intensity increases gently with excitation energy until the threshold. The lasing threshold for $605.0 \mathrm{~nm}, 611.9 \mathrm{~nm}$, and $677.1 \mathrm{~nm}$ lasing is $11.8 \mu \mathrm{J} / \mathrm{pulse}$, $12.6 \mu \mathrm{J} /$ pulse, and $14.8 \mu \mathrm{J} /$ pulse, respectively. The lasing threshold for $677.1 \mathrm{~nm}$ lasing is higher than that of $605 \mathrm{~nm}$ lasing because the net-gain decreases intensely at the gain edge even though it operates at a lower diffraction order $[30,33,34]$. The net-gain for the $611.9 \mathrm{~nm}$ lasing is smaller than the $605.0 \mathrm{~nm}$ lasing, and the lasing threshold is thus higher.

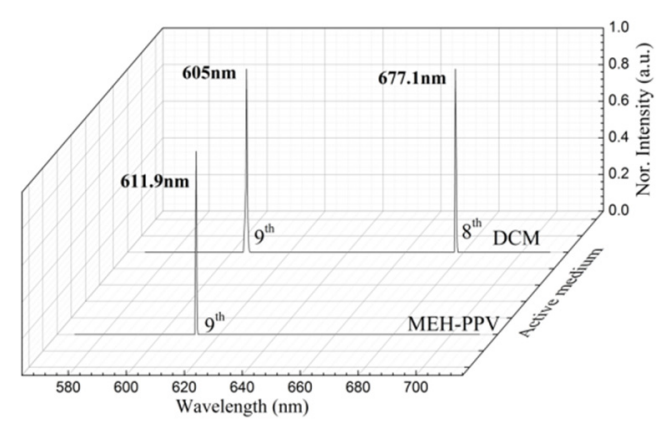

(a)

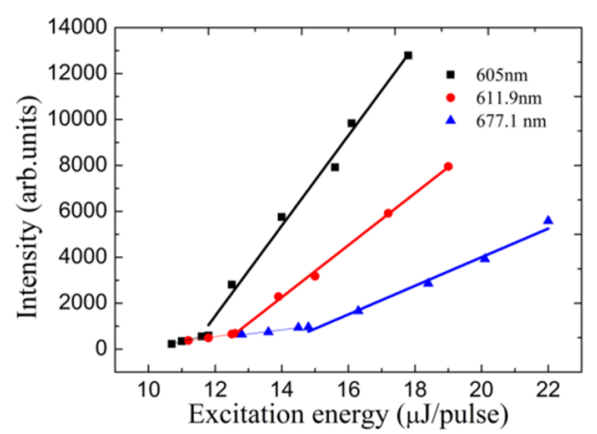

(b)

Figure 5. Tri-wavelength lasing property for the dye-doped HPDLC DFB laser at $20{ }^{\circ} \mathrm{C}$. (a) Tri-wavelength lasing spectra of the dye-doped holographic polymer-dispersed liquid crystal (HPDLC)-distributed feedback (DFB) laser. The grating period was $1.72 \mu \mathrm{m}$ for the tri-wavelength lasing operation. (b) Dependence of output emission intensity to excitation energy for different wavelength.

\subsection{Tuning Property for the Tri-Wavelength Lasing HPDLC DFB Laser by Elevating Temperature}

Thanks to the positive nematic LC layer in the HPDLC holographic grating, the lasing tunes by the variation of operation temperature. The molecular configuration transforms from the nematic phase state to the isotropic phase state when temperature changes (N-I transition) [35]. The N-I transition temperature for the LC TEB-30A used in this work is $61.3^{\circ} \mathrm{C}$ (DSC Q2000). The sample was in full contact with a hotplate during optical pumping, and the sample temperature was controlled by a thermostat from 20 to $70^{\circ} \mathrm{C}$. Figure 6 illustrates the tuning property for the tri-wavelength lasing HPDLC DFB laser by elevating temperature. Narrow peaks are observed in every lasing wavelength, 
which indicates that laser action is preserved. The center wavelength shifts to the high energy range with temperature. The reason is that the mean refractive index of LC decreases with temperature, which leads to the decrease in the mode effective refractive index according to Equation (2). The center wavelength for the $605.0 \mathrm{~nm}$ lasing changes from 605.0 to $596.5 \mathrm{~nm}$ when the temperature increases from 20 to $70{ }^{\circ} \mathrm{C}$, which corresponds to an $8.5 \mathrm{~nm}$ blue shift. As for the other lasing from DCM, the center wavelength changes from 677.1 to $669.5 \mathrm{~nm}$ when the temperature increases from 20 to $70{ }^{\circ} \mathrm{C}$, which corresponds to a $7.6 \mathrm{~nm}$ blue shift. As for the lasing from MEH-PPV, the center wavelength varies from 611.9 to $603.0 \mathrm{~nm}$ with the temperature increasing from 20 to $70{ }^{\circ} \mathrm{C}$, which corresponds to an $8.9 \mathrm{~nm}$ blue-shift as shown in Figure 6a. In a word, the temperature-dependence tuning range for the tri-wavelength dye-doped HPDLC DFB laser is as high as $8 \mathrm{~nm}$. Figure $6 \mathrm{~b}$ shows the dependence of a working lasing threshold with an operation temperature. The lasing threshold for the $605 \mathrm{~nm}$ lasing increases from 11.8 to $14.9 \mu \mathrm{J} /$ pulse when the temperature increases from 20 to $70{ }^{\circ} \mathrm{C}$. As for the other lasing from DCM, the lasing threshold increases from 14.8 to $19.4 \mu \mathrm{J} /$ pulse when the temperature increases from 20 to $70{ }^{\circ} \mathrm{C}$. As for the lasing from MEH-PPV, the lasing threshold increases from 12.6 to $15.3 \mu \mathrm{J} /$ pulse with the temperature increasing from 20 to $70{ }^{\circ} \mathrm{C}$. The reason for the increase in the lasing threshold is that the coupling decreases with the elevating temperature according to the coupled wave theory [24]. The laser device, which is lightweight, smart, and cost-effective and enjoys one-step holography fabrication, tri-wavelength operation, and temperature-dependent tunability, is promising in the fields of sensing and spectroscopy [6].

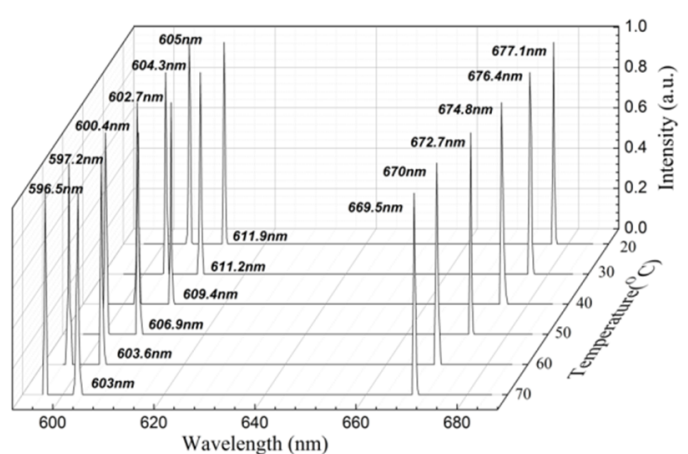

(a)

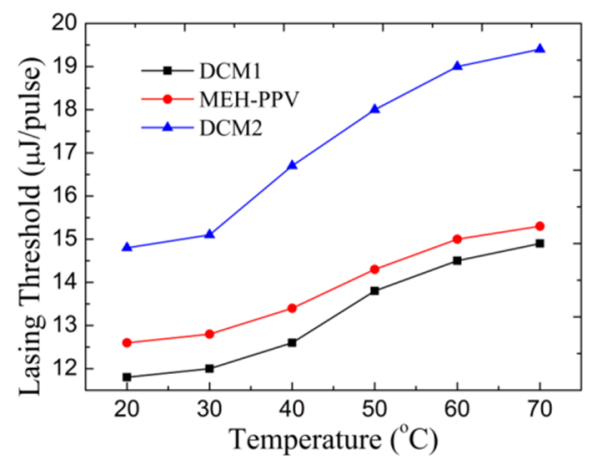

(b)

Figure 6. Tuning property for the tri-wavelength lasing HPDLC DFB laser by elevating temperature. (a) Dependence of the lasing spectra to operation temperature. (b) The working lasing threshold as a function of the operation temperature.

\section{Conclusions}

In summary, organic tri-wavelength lasing was achieved from a holographic polymer-dispersed liquid crystal and a distributed feedback laser with a semiconducting polymer poly[-methoxy-5-(2'-ethyl-hexyloxy)-1,4-phenylene-vinylene] (MEH-PPV) and laser dye [4-(dicyanomethylene)-2-methyl-6-(p-dimethylaminostyryl)-4H-pyran] (DCM) by one-step holography, which centered at $605.0 \mathrm{~nm}$ (DCM), $611.9 \mathrm{~nm}$ (MEH-PPV), and $677.1 \mathrm{~nm}$ (DCM). The lasing emission from the 9th order HPDLC DFB laser with MEH-PPV as an active medium was also investigated, which showed excellent s-polarization characterization. The grating period was selected at $1.72 \mu \mathrm{m}$ to achieve tri-wavelength lasing. The diffraction order is 9th and 8th for the dual-wavelength lasing with DCM as the active medium. The temperature-dependence tuning range for the tri-wavelength dye-doped HPDLC DFB laser was as high as $8 \mathrm{~nm}$. We believe that the results of this work provide a method for constructing the compact and cost-effective solid-state smart laser systems, which may find applications in scientific and applied research where multi-wavelength radiation is required. 
Acknowledgments: This work is supported by the National Natural Science Foundation of China (61377032, 61378075).

Author Contributions: Minghuan Liu conceived and designed the experiments; Minghuan Liu performed the experiments; Minghuan Liu, Qidong Wang, Quanquan Mu and Zhaoliang Cao analyzed the data; Shaoxin Wang, Yonggang Liu, Zenghui Peng, and Li Xuan contributed reagents/materials/analysis tools; Minghuan Liu wrote the paper.

Conflicts of Interest: The authors declare no conflict of interest.

\section{References}

1. Llobera, A.; Juvert, J.; Gonzalez-Fernandez, A.; Ibarlucea, B.; Carregal-Romero, E.; Buttgenbach, S.; Fernandez-Sanchez, C. Biofunctionalized all-polymer photonic lab on a chip with integrated solid-state light emitter. Light Sci. Appl. 2015, 4, e271. [CrossRef]

2. Samuel, I.D.W.; Turnbull, G.A. Organic semiconductor lasers. Chem. Rev. 2007, 107, 1272-1295. [CrossRef] [PubMed]

3. Chenais, S.; Forget, S. Recent advances in solid-state organic lasers. Polym. Int. 2012, 61, 390-406. [CrossRef]

4. Grivas, C.; Pollnau, M. Organic solid-state integrated amplifiers and lasers. Laser Photonics Rev. 2012, 6, 419-462. [CrossRef]

5. Zhang, Y.F.; Forrest, S.R. Existence of continuous-wave threshold for organic semiconductor lasers. Phys. Rev. B 2011, 84, 241301. [CrossRef]

6. Woggon, T.; Klinkhammer, S.; Lemmer, U. Compact spectroscopy system based on tunable organic semiconductor lasers. Appl. Phys. B Lasers Opt. 2010, 99, 47-51. [CrossRef]

7. Mhibik, O.; Forget, S.; Ott, D.; Venus, G.; Divliansky, I.; Glebov, L.; Chenais, S. An ultra-narrow linewidth solution-processed organic laser. Light Sci. Appl. 2016, 5, e16026. [CrossRef]

8. Vannahme, C.; Dufva, M.; Kristensen, A. High frame rate multi-resonance imaging refractometry with distributed feedback dye laser sensor. Light Sci. Appl. 2015, 4, e269. [CrossRef]

9. Bulovic, V.; Kozlov, V.G.; Khalfin, V.B.; Forrest, S.R. Transform-limited, narrow-linewidth lasing action in organic semiconductor microcavities. Science 1998, 279, 553-555. [CrossRef] [PubMed]

10. Tessler, N.; Denton, G.J.; Friend, R.H. Lasing from conjugated-polymer microcavities. Nature 1996, 382, 695-697. [CrossRef]

11. Whitworth, G.L.; Zhang, S.; Stevenson, J.R.Y.; Ebenhoch, B.; Samuel, I.D.W.; Turnbull, G.A. Solvent immersion nanoimprint lithography of fluorescent conjugated polymers. Appl. Phys. Lett. 2015, 107, 163301. [CrossRef]

12. Vasdekis, A.E.; Tsiminis, G.; Ribierre, J.C.; O’Faolain, L.; Krauss, T.F.; Turnbull, G.A.; Samuel, I.D.W. Diode pumped distributed Bragg reflector lasers based on a dye-to-polymer energy transfer blend. Opt. Express 2006, 14, 9211-9216. [CrossRef] [PubMed]

13. Hermann, S.; Shallcross, R.C.; Meerholz, K. Simple Fabrication of an Organic Laser by Microcontact Molding of a Distributed Feedback Grating. Adv. Mater. 2014, 26, 6019-6024. [CrossRef] [PubMed]

14. Bunning, T.J.; Natarajan, L.V.; Tondiglia, V.P.; Sutherland, R.L. Holographic polymer-dispersed liquid crystals (H-PDLCs). Annu. Rev. Mater. Sci. 2000, 30, 83-115. [CrossRef]

15. Clark, J.; Lanzani, G. Organic photonics for communications. Nat. Photonics 2010, 4, 438-446. [CrossRef]

16. Vannahme, C.; Klinkhammer, S.; Lemmer, U.; Mappes, T. Plastic lab-on-a-chip for fluorescence excitation with integrated organic semiconductor lasers. Opt. Express 2011, 19, 8179-8186. [CrossRef] [PubMed]

17. Diao, Z.H.; Deng, S.P.; Huang, W.B.; Xuan, L.; Hu, L.F.; Liu, Y.G.; Ma, J. Organic dual-wavelength distributed feedback laser empowered by dye-doped holography. J. Mater. Chem. 2012, 22, 23331-23334. [CrossRef]

18. Zhang, G.Y.; Liu, L.J.; Liu, M.H.; Liu, Y.G.; Peng, Z.H.; Yao, L.S.; Wang, Q.D.; Wang, S.X.; Cao, Z.L.; Ma, J.; et al. Tunable surface-emitting dual-wavelength laser from a blended gain layer with an external holographic grating feedback structure. Opt. Mater. Express 2016, 6, 3320-3329. [CrossRef]

19. Liu, M.H.; Liu, Y.G.; Zhang, G.Y.; Peng, Z.H.; Li, D.Y.; Ma, J.; Xuan, L. Organic holographic polymer dispersed liquid crystal distributed feedback laser from different diffraction orders. J. Phys. D Appl. Phys. 2016, 49, 465102. [CrossRef]

20. Huang, W.; Diao, Z.; Liu, Y.; Peng, Z.; Yang, C.; Ma, J.; Xuan, L. Distributed feedback polymer laser with an external feedback structure fabricated by holographic polymerization technique. Org. Electron. 2012, 13, 2307-2311. [CrossRef] 
21. Tammer, M.; Monkman, A.P. Measurement of the anisotropic refractive indices of spin cast thin poly(2-methoxy-5-(2'-ethyl-hexyloxy)-p-phenylenevinylene) (MEH-PPV) films. Adv. Mater. 2002, 14, 210-212. [CrossRef]

22. Vannahme, C.; Klinkhammer, S.; Christiansen, M.B.; Kolew, A.; Kristensen, A.; Lemmer, U.; Mappes, T. All-polymer organic semiconductor laser chips: Parallel fabrication and encapsulation. Opt. Express 2010, 18, 24881-24887. [CrossRef] [PubMed]

23. Samuel, I.D.W.; Namdas, E.B.; Turnbull, G.A. How to recognize lasing. Nat. Photonics 2009, 3, 546-549. [CrossRef]

24. Kogelnik, H.; Shank, C.V. Coupled-Wave Theory of Distributed Feedback Lasers. J. Appl. Phys. 1972, 43, 2327-2335. [CrossRef]

25. Steinle, T.; Kumar, V.; Floess, M.; Steinmann, A.; Marangoni, M.; Koch, C.; Wege, C.; Cerullo, G.; Giessen, H. Synchronization-free all-solid-state laser system for stimulated Raman scattering microscopy. Light Sci. Appl. 2016, 5, e16149. [CrossRef]

26. Liang, S. T. Physical Optics; Chapter 1; Electronics Industry: Beijing, China, 2011.

27. Ziebarth, J.M.; McGehee, M.D. Measuring the refractive indices of conjugated polymer films with Bragg grating outcouplers. Appl. Phys. Lett. 2003, 83, 5092-5094. [CrossRef]

28. Turnbull, G.A.; Andrew, P.; Barnes, W.L.; Samuel, I.D.W. Photonic mode dispersion of a two-dimensional distributed feedback polymer laser. Phys. Rev. B 2003, 67, 165107. [CrossRef]

29. Lozano, G.; Rodriguez, S.R.K.; Verschuuren, M.A.; Rivas, J.G. Metallic nanostructures for efficient LED lighting. Light Sci. Appl. 2016, 5, e16080. [CrossRef]

30. Kapon, E.; Hardy, A.; Katzir, A. The Effect of Complex Coupling-Coefficients on Distributed Feedback Lasers. IEEE J. Quantum Electron. 1982, 18, 66-71. [CrossRef]

31. Schneider, D.; Rabe, T.; Riedl, T.; Dobbertin, T.; Kroger, M.; Becker, E.; Johannes, H.H.; Kowalsky, W.; Weimann, T.; Wang, J.; et al. Ultrawide tuning range in doped organic solid-state lasers. Appl. Phys. Lett. 2004, 85, 1886-1888. [CrossRef]

32. Criante, L.; Lucchetta, D.E.; Vita, F.; Castagna, R.; Simoni, F. Distributed feedback all-organic microlaser based on holographic polymer dispersed liquid crystals. Appl. Phys. Lett. 2009, 94, 111114. [CrossRef]

33. Cornil, J.; Beljonne, D.; Calbert, J.P.; Bredas, J.L. Interchain interactions in organic pi-conjugated materials: Impact on electronic structure, optical response, and charge transport. Adv. Mater. 2001, 13, 1053-1067. [CrossRef]

34. Riechel, S.; Lemmer, U.; Feldmann, J.; Benstem, T.; Kowalsky, W.; Scherf, U.; Gombert, A.; Wittwer, V. Laser modes in organic solid-state distributed feedback lasers. Appl. Phys. B Lasers Opt. 2000, 71, 897-900. [CrossRef]

35. Kakiuchida, H.; Tazawa, M.; Yoshimura, K.; Ogiwara, A. Thermal control of transmittance/diffraction states of holographic structures composed of polymer and liquid crystal phases. Sol. Energy Mater. Sol. Cells 2010, 94, 1747-1752. [CrossRef]

(C) 2017 by the authors. Licensee MDPI, Basel, Switzerland. This article is an open access article distributed under the terms and conditions of the Creative Commons Attribution (CC BY) license (http:// creativecommons.org/licenses/by/4.0/). 